\title{
Brain Tumor Epilepsy Seizure Identification using Multi- Wavelet Transform, Neural Network and Clinical Diagnosis Data
}

\author{
Sharanreddy. M \\ Dept, EEE \\ P D A College of Engineering \\ Gulbarga, India
}

\author{
P.K.Kulkarni, PhD. \\ Dept, EEE \\ P D A College of Engineering \\ Gulbarga, India
}

\begin{abstract}
In the last couple of years, the EEG signal analysis was focused on epilepsy seizure detection. Epilepsy is a common chronic neurological disorder; they are result of transient and unexpected electrical disturbance of the brain. Epilepsy seizures also a symptom of brain tumor existence, 30\% patients with brain tumor are affected with epilepsy seizure. This paper proposes a two level brain tumor epilepsy seizure identification method that combines bio-medical engineering techniques and clinical diagnosis data. First level classify the given EEG signal in to normal and epilepsy seizure, based on the first level input second level identifies the epilepsy seizure signal is from brain tumor or other neural disorders. Proposed method uses multi wavelet transform for feature extraction, in which EEG signal is decompose in to sub-bands. Irregularities present in the EEG signal are measured by using the approximate entropy. Feed forward neural network is used to classify input EEG signal as normal and brain tumor epilepsy signal. Obtained results are promising with first level epilepsy seizure identification accuracy of $93 \%$.
\end{abstract}

\section{General Terms}

Brain Tumor Epilepsy Seizure Identification. Clinical Diagnosis Data.

\section{Keywords}

Artificial Intelligence (AI), Brain tumor, Clinical Diagnosis, Epilepsy Seizure, Electroencephalogram (EEG), Multiwavelet transforms (MWT), Neural Network (NN).

\section{INTRODUCTION}

Brain electrical activities are recorded using EEG clinical test. EEG signal rhythmic activities represent the postsynaptic potentials of vertically oriented pyramidal cells of the cerebral cortex and characterized by frequency. EEG test carried out to monitor condition of brain. EEG test results are useful in identifying central nervous system disorders, like epilepsy seizure, brain tumors, cerebrovascular disorders, metabolic and toxic encephalopathies [1].

\subsection{EEG abnormalities associated with Brain Tumor EEG with Epilepsy seizures}

The EEG is most useful in evaluating patients with suspected epilepsy. The presence of electrographic seizure activity, i.e. of abnormal, repetitive, rhythmic activity having an abrupt onset and termination, clearly establishes the diagnosis. EEG findings contribute to the multi-dimensional diagnosis of epilepsy, in terms of whether the seizure disorder is focal or generalized, idiopathic or symptomatic, or part of a specific epilepsy syndrome.
Tumor tissue is electrically silent (with the possible exception of tumors containing neuronal elements, such as gangliogliomas), hence EEG changes observed with brain tumors are mainly from disturbances in bordering brain parenchyma. For this reason, EEG localization often is misleading, although lateralization is generally reliable.

Slowly growing gliomas such as oligodendrogliomas and fibrillary astrocytomas (excluding tumors of deep structures) often can be distinguished from the more rapidly growing anaplastic astrocytoma and glioblastoma multiforme. With gliomas tumors, the abnormalities tend to be localized within the theta range. Gliomas commonly cause seizures and epileptiform activity appear before slow waves. Later, delta wave appears intermittently with $2-3 \mathrm{~Hz}$ frequency. Still later, focal that polymorphic delta activity (PDA) becomes persistent.

Spikes, sharp waves, or spike-wave complexes occurring with consistent localization are observed uncommonly in the early course of tumors. However, they are more common either as early findings of slowly growing neoplasms associated with seizures.

Focal delta activity is the classic electrographic sign of a local disturbance in cerebral function. A structural lesion is strongly suggested if the delta activity is continuously present. EEG waves show variations in amplitude, duration, morphology (polymorphic) and persists changes in physiologic states, such as sleep or alerting procedures. When focal delta is found without a corresponding imaging abnormality, it is usually in case of acute seizures (especially postictally), nonhemorrhagic infarction or trauma.

EEG patterns seen in epileptic patients are either nonspecific or specific. Nonspecific patterns can be seen in many conditions besides epilepsy, such as stroke, head trauma and brain tumor. Slowing of the brain waves is an example of a nonspecific pattern, commonly seen in epileptic patients. The International Federation of Societies for Electroencephalography and Clinical Neurophysiology says that specific patterns indicating a tendency toward seizures or epilepsy waves include spikes, spike-and-wave discharges and sharp waves $[1,5]$.

\subsubsection{Spikes}

Epileptic seizures are caused by a group of neurons firing simultaneously. These are visible on EEG as sudden burst of electricity or "spikes" that are easy to discriminate from the background activity. The duration of a spike ranges from 20 to 70 milliseconds. 


\subsubsection{Sharp Waves}

Just like spikes, sharp waves can easily be distinguished from the background activity. They have a pointed peak and duration of about 70 to 200 milliseconds. Spikes and sharp waves seen in a local brain area is a sign of a partial seizure as compared to generalized seizure.

\subsubsection{Spike and Slow Wave Complex}

Spike-and-slow wave pattern consists of a spike that is followed by a slow wave. Typically, the slow wave is of higher amplitude than the spike. Multiple spike-and-slowwave complexes can also be seen. They are just like spikeand-slow-wave patters except that one or more slow waves are accompanied by at least two spikes. If spike-and-wave patterns can be seen in a widespread area in both cerebral hemispheres, especially if they begin in both hemispheres at the same time, a patient is likely to have generalized epilepsy. A $3 \mathrm{~Hz}$ spike-and-wave activity is specific to an epilepsy type called petit mal. Fig. 1 shows the EEG signal with epilepsy seizure from gliomas brain tumor patient.

When a person has epilepsy, the location and exact pattern of the abnormal brain waves show what type of epilepsy or seizures the person has. An important observation is that in many people with epilepsy, the EEG may appear completely normal between seizures. The EEG shows delta waves or too many theta waves in adults who are awake.

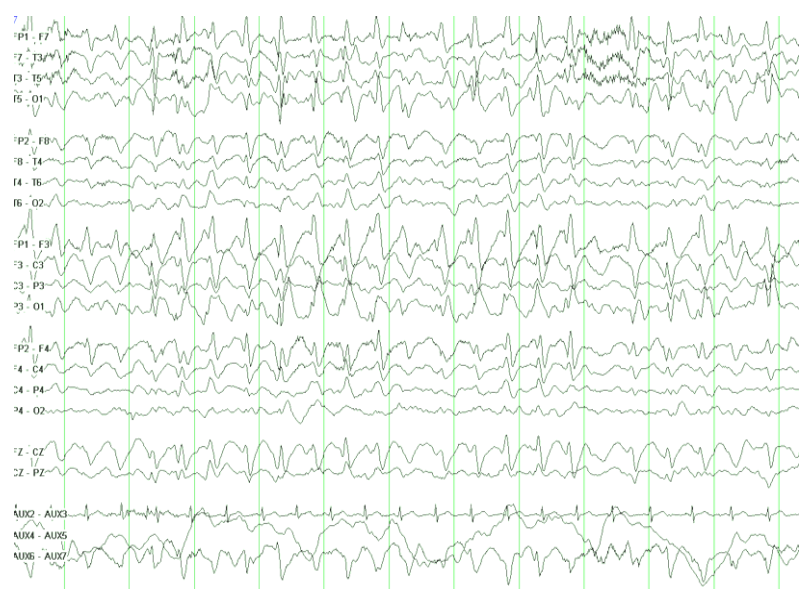

Fig 1: Spike and slow wave pattern observed in the gliomas brain tumor patient

\subsection{Clinical Diagnosis}

Clinical diagnosis plays an important role in identifying the diseases. Making a diagnosis is the pivotal cognitive activity of every practicing doctor. A correct diagnosis leads to appropriate treatment. With the high cost of health care, increased patient awareness, medico-legal and insurance pressures, every doctor must be empathic, accountable and cost-effective in patient care.

Diagnosing the disease depends on capacity to knowledge and experience accumulated over years of practice. Hence by referring the literature [8], consulting Neurologist and brain tumor patients, a set of 11 questions are pre-paired. These questions differentiate the brain tumor and other neural disorders and help in identifying brain tumor epilepsy seizure EEG signal. Theses question are to be answered by patient at the time of EEG signal classification. Following are the questions which need to be answered by patient.

\subsubsection{Morning Headaches}

About half of all patients with brain tumors experience headache. Tumor builds the pressure in the skull, which results headaches. Headache worst early in the morning (or during the night) and disappear soon after the person gets up. They are often mild at first, but over days to weeks become gradually more severe, frequent and last longer each time and eventually become almost constant. They are worsened by bending over and can cause nausea and vomiting.

The most common type of headache in people with brain tumors is an ordinary tension-type headache - usually described as a dull ache, a feeling of pressure or similar to a headache caused by sinusitis. It is usually on both sides of the head, but may be worse on the side of the tumor. Some people experience migraine-like headaches, but this is less common. Some patients experience a mixture of headache types. Some people describe the headache as a throbbing pain or shooting pain.

\subsubsection{Vomiting / Weakness}

Nausea and vomiting are most commonly the result of increased intracranial pressure due to tumor growth. Vomiting, especially in the morning and without nausea, can be a symptom of a brain tumor. Adults with a brain tumor may experience weakness on one side of the body. They may become suddenly "clumsy" -- losing balance or walking into walls or stumbling. An abnormal gait may also be present. Coordinated movements may become difficult.

\subsubsection{Change in the Taste / Hearing or Smell}

Some brain tumors can cause hearing disturbances that are difficult to ignore. Hearing disturbances can include one-sided hearing loss and ringing in the ears. Brain tumors patients may not able differentiate and feel change in taste and smell.

\subsubsection{Change in Personality / Mood / Behavioral}

Adults with brain tumors sometimes experience personality changes that are frustrating and can definitely interrupt daily living activities. Laughing at things that are not humorous, sudden increased interest in sex, temper tantrums, paranoia, and social decline are just a few of the possible personality changes that one may experience if they have a brain tumor. In contrast, personality changes can also mean an exaggeration of normal characteristics.

\subsubsection{Double Vision / Decreased in Vision}

Some tumors create pressure on the optic nerve, which affect the visual pathways; hence vision problem may occur in brain tumor patients. Problems with vision can include seeing flashing lights, blurring, tunnel vision and floaters. This could happen in one or both eyes.

\subsubsection{Hand/Head tremor or Numbness}

Hand or Head tremor is an unintentional and uncontrollable rhythmic movement of one part of your body. It is usually the result of a problem in the part of the brain that controls your muscles. Although tremors are not always serious, in some cases they may indicate a serious disorder. Brain tumor patients may shown numbness, it is an abnormal feeling or loss of sensation on the surface of the skin, including skin rash, dry skin, cool or mottled skin, burning pain, increased sensitivity to touch.

\subsubsection{Speech Changes}

Tumor patients show signs of slurring of the words or slow speech. A person with a brain tumor may say things that make very little sense, despite efforts to communicate with the 
correct words. Sentences may have words in the incorrect order or even include words that have no relevance. This lack of effective communication can be a frustrating symptom for people with brain tumors.

\subsubsection{Difficulty in Writing or Reading}

Slow processing speed of the brain can be a symptom of a brain tumor. Brain tumor patients takes longer time to complete tasks than it usually does, this isn't related to fatigue or lack of motivation. These are tasks that require thinking like simple math, writing sentences, setting up a chess board, or following a recipe. People with brain tumors may find it takes great effort to complete the most basic task.

\subsubsection{Feel pain, pressure or temperatures}

An increase of pressure in the skull is called raises intracranial pressure (ICP), this pressure makes patients sick and feel pain (Grade II brain tumors), it may be worse in the morning.

\subsubsection{Confusion or Memory loss}

Brain tumors can cause problems with a person's thinking (cognitive function), which includes ability to remember, learn, recognize things, solve problems, reason and make decisions. At the time of diagnosis, a person may already have mild problems with cognitive function, especially if they have a rapidly growing, high-grade tumor. However, it is unusual for these to be the only symptoms that lead to the diagnosis of brain tumor. These symptoms do not help identify the type of brain tumor.

Literature [9] shows that, no researcher made an attempt to develop an automated tool which analyses the EEG signals and gives information of existence of brain tumor epilepsy seizure using clinical diagnosis data. This paper proposes a automated tool, which analyses EEG signal for detection of brain tumor epilepsy.

\section{PROPOSED METHODOLOGY}

Proposed method implanted in two levels. First level classifies the given EEG signal in to normal and epilepsy seizure. Based on the first level input, second level identifies the epilepsy signal is from brain tumor or some other neural disorders. Fig.2. Shows block diagram shows flow of proposed methodology.

\subsection{Database used for proposed work}

For Brain tumor epilepsy seizure identification 150 samples of EEG data collected from 10 subjects (7 males, ages 23- 52; and 3 females, ages 35-49). These patients identified with brain tumor and EEG signal are recorded using continuous monitoring system. Out of which 125 data of each diseases used to train the neural network and remaining data are used for testing. All signals were sampled at 256 samples per second with 16-bit resolution. The International 10-20 system of EEG electrode positions and nomenclature was used for these recordings.

\subsection{First Level}

In the first level EEG signal without any art affect is given as an input to MWT, the EEG signal is decomposed and the irregularities of the signal are determined by using the ApE process. The ApE output is trained by using Feed Forward Neural Network (FFNN), trained neural network classify the input signal as normal and epilepsy seizure.

\subsubsection{Multi Wavelet Transform Decomposition}

In MWT decomposition, the input signal is denoted as $x(n)$, decomposed low pass filter outputs are denoted as $A_{1}, A_{2}, A_{3}, A_{4}$ and $A_{5}$ and the decomposed high pass filter outputs are denoted as $D_{1}, D_{2}, D_{3}, D_{4}$ and $D_{5}$. Fig.3. shows the decomposition structure of MWT. The decomposition of MWT is calculated by using the below formulas.

The decomposition of low frequency component is calculated as, $A_{i-1}=\sum_{k} H_{k} A_{i, 2 k+n}$

The decomposition of high frequency component is calculated as, $D_{i-1}=\sum_{k} G_{k} D_{i, 2 k+n}$

\subsubsection{Approximate Entropy Method}

Approximate entropy (ApE) is a technique used to quantify the amount of regularity and the unpredictability of fluctuations over time-series data. The output of $\mathrm{ApE}$ is denoted as $A D_{1}, A D_{2}, A D_{3}, A D_{4}, A D_{5}$ and $A A_{5}$.

The irregularities of the EEG signal are calculated by following the steps.

1. Calculate $\mathrm{N}$ data points from the signal i.e. $n=\left[n_{(1)}, n_{(2)}, \ldots \ldots \ldots \ldots n_{(N)}\right]$

2. Fix window length $m$ and tolerance $r r$

3. Form a sequence of vector $x(1), x(2), \ldots \ldots x(N-m+1), \mathrm{m}$ dimensional vectors are defined by $x(i)=[u(i), u(i+1), \ldots \ldots . . ., u(i+m-1)] \quad$ for $i=1,2$, ..... N- $m+1$

4. Using sequence $x(1), x(2), \ldots . . x(N-m+1)$ to construct for each $i \quad 1 \leq i \leq N-m+1$. Calculate the absolute difference between their respective scalar components i.e.,

$$
\begin{aligned}
& d[x(i), x(j)]=\max _{k=1,2 \ldots . . m}|u(i+k-1)-u(j+k-1)| \leq r r \\
& \text { Calculate, } \quad C_{i}^{m}(r r)=\frac{d[x(i), x(j)]}{(N-m+1)}
\end{aligned}
$$

5. Calculate natural algorithm for each value of $C_{i}^{m}(r r)$ and average it over $i$ $\phi^{m}(r r)=(N-m+1) \sum_{i=1}^{-1} \operatorname{In} C_{i}^{m}(r r)$

6. Calculate $C_{i}^{m+1}(r r)$ and $\phi^{m+1}(r r)$ for increasing the $\mathrm{m}$ up to its fixed value, ApE calculated by below formula,

$$
A p E=\phi^{m}(r r)-\phi^{m+1}(r r)
$$

The irregularities of signal depend on the ApE value. ApE value for each sub-signal of the decomposed data with MWT is calculated to form a feature vector. These ApE value is then applied as input to the neural network and the training dataset is generated. 


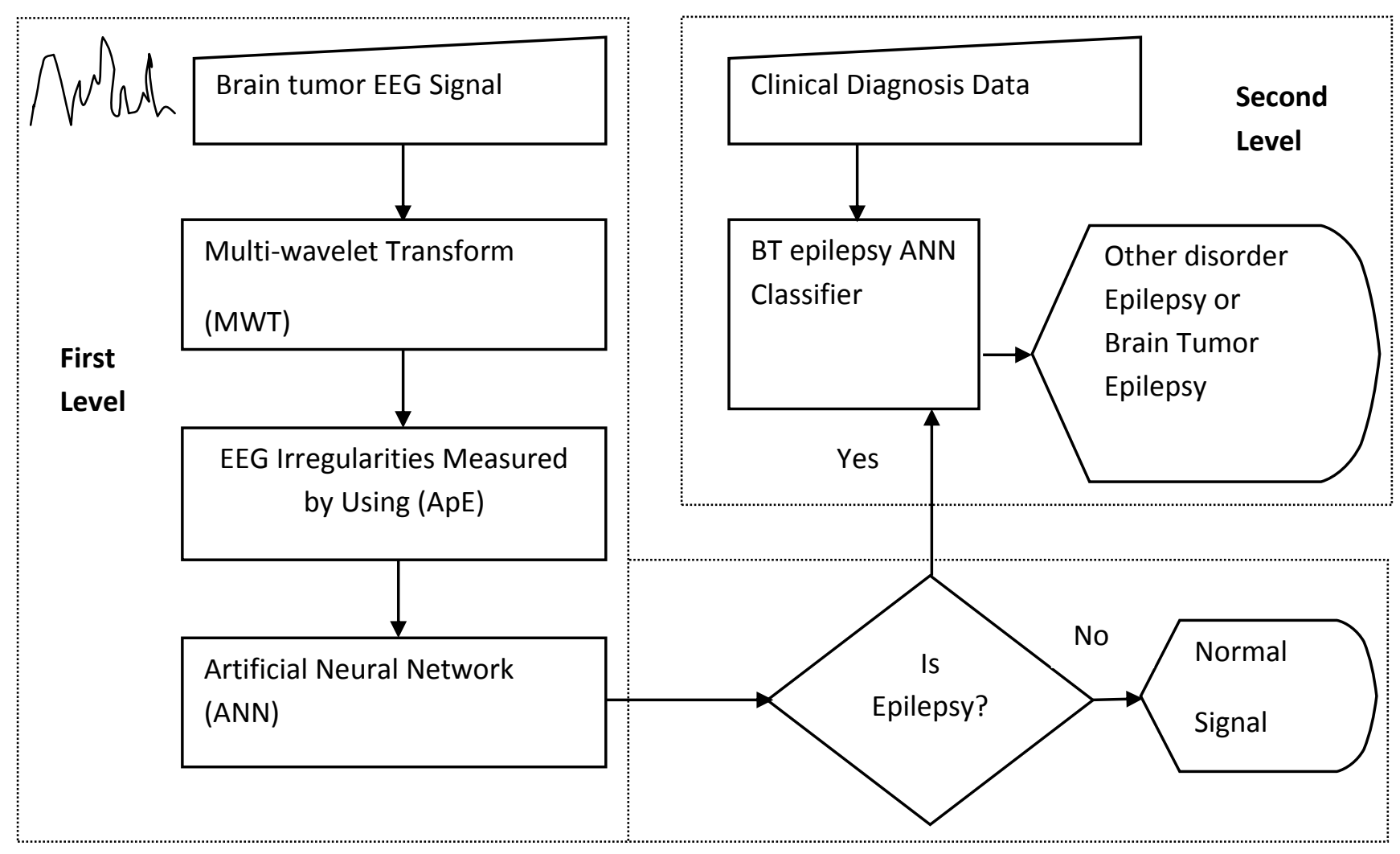

Fig 2: Block diagram of proposed Methodology

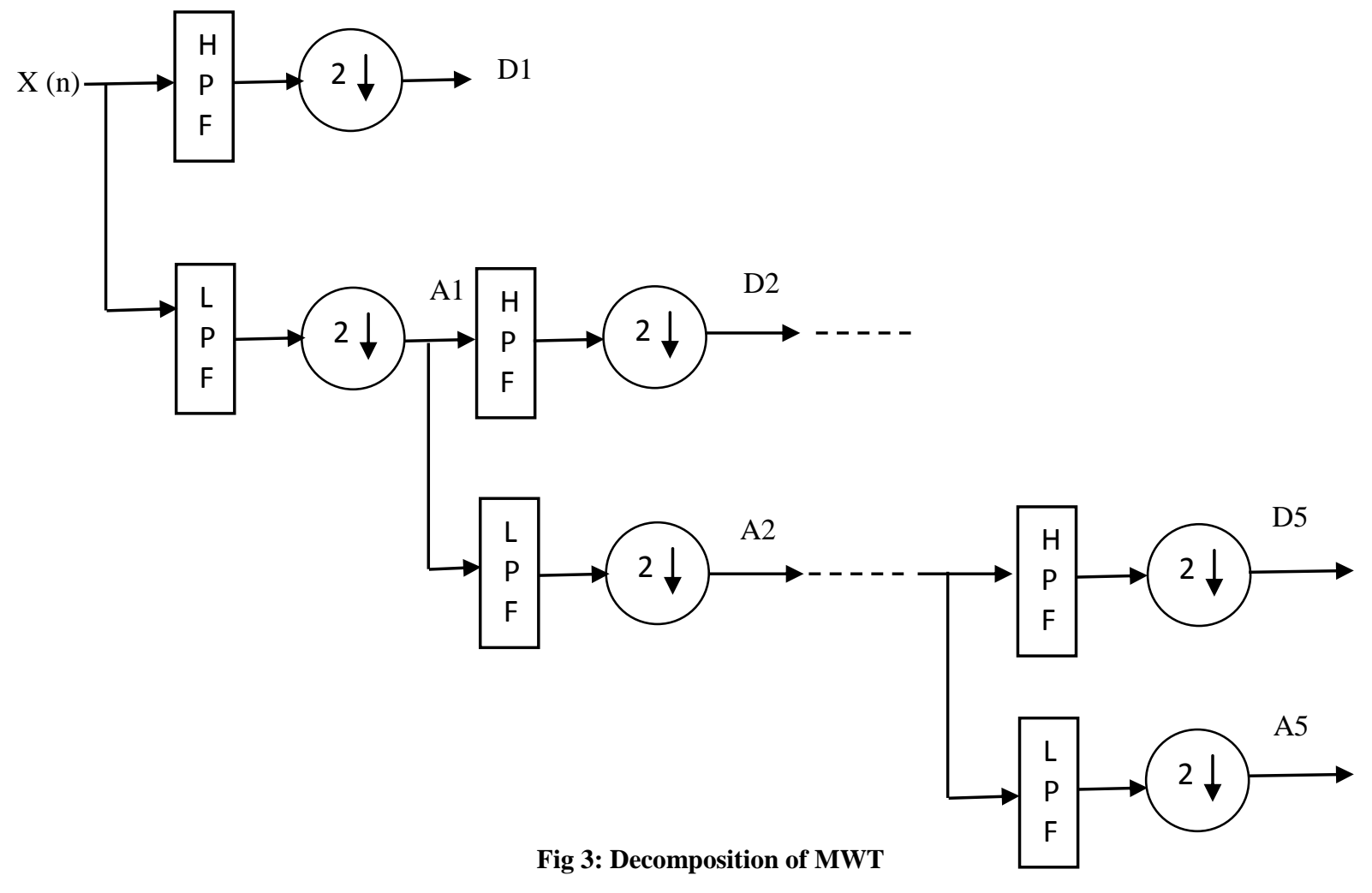




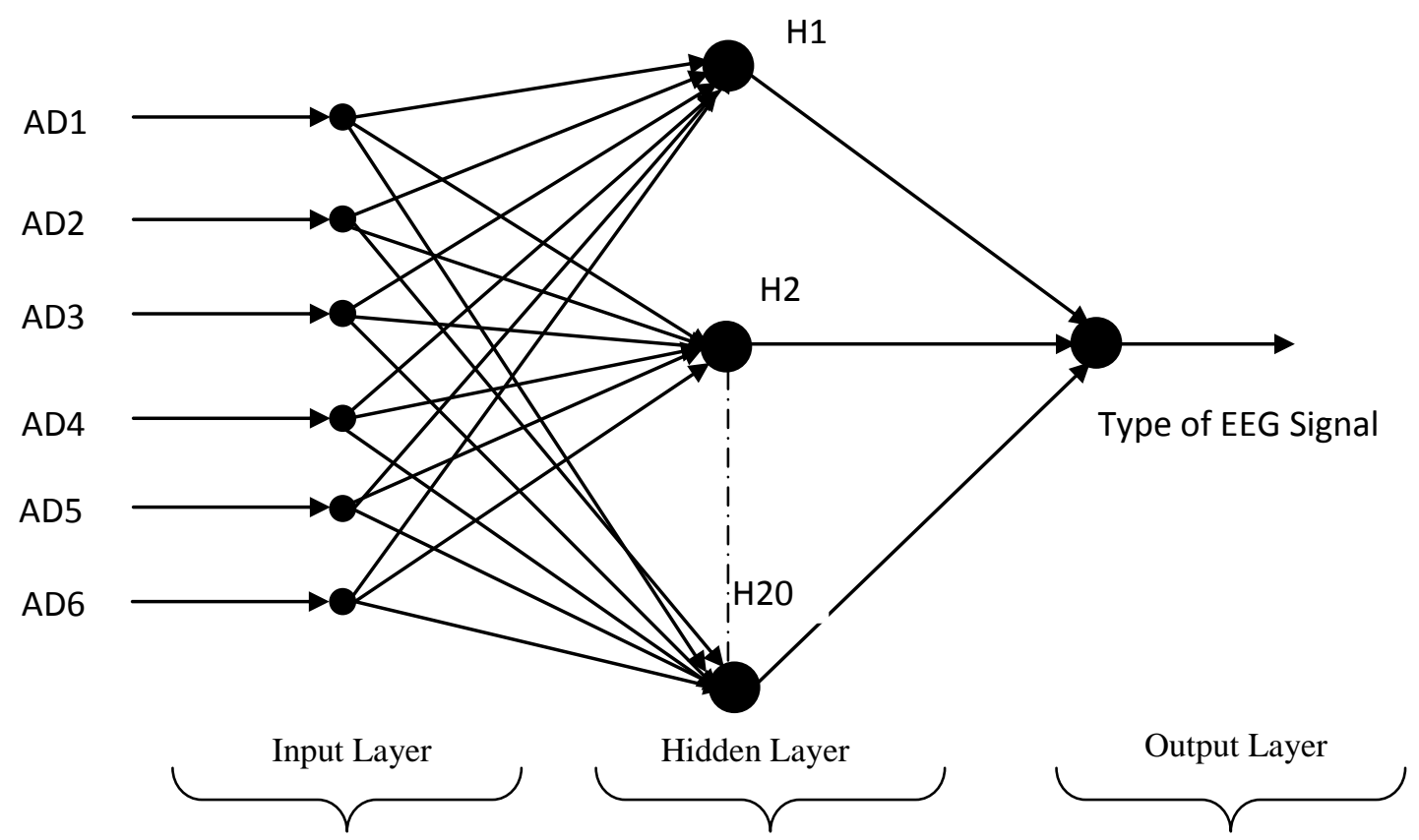

Figure 4: Neural network training structure

\subsubsection{Training of Neural Network}

In the present work, a feed-forward neural network (FFNN) is used for identifying the types of EEG signal. FFNN consists of three layers namely input layer, hidden layer and output layer. The input to input layers of neural network are $A D_{1}, A D_{2}, A D_{3}, A D_{4}, A D_{5}$ and $A A_{5}$. The $n$ numbers of hidden layers and $H_{1}, H_{2}, \ldots \ldots \ldots \ldots . . . . H_{n}$ are nodes of hidden layer; the neural network process takes place in this hidden layer. The training of the neural network is performed by back propagation algorithm. The output of neural network is used to determine the types of EEG signal. Fig.4. show the neural network training structure. The multi-wavelet output is trained and the training dataset is generated for primary brain tumor detection. The weight between input and hidden layer is denoted as $W_{1}$, the weight between hidden and output layer is denoted as $W_{2}$. The weight adjustment depends on the output requirement.

The formula for weight adjustment between the layers is

$$
W_{j i}(n+1)=W_{j i}(n)+\Delta W_{j i}(n) .
$$

The neural network output is calculated by using the formula

$$
\sum_{j=i=1}^{n} W_{j i} A D
$$

Once the training process is completed, then, the network is stored for testing. In the testing phase, an input EEG signal applied to trained network, which classify the given input signal is of epilepsy or normal.

\subsection{Second Level}

Using first level input, second level identifies the epilepsy seizure is from brain tumor or some other neural disorders. Fig.5. shows the clinical data questions, which need to answer by patient at the time of EEG test. These questions are given as input to brain tumor epilepsy ANN classifier.

\subsubsection{Brain Tumor epilepsy ANN Classifier}

Brain tumor epilepsy ANN classifier uses feed-forward neural network (FFNN) to identify the source of epilepsy seizure. Training dataset is generated using clinical diagnosis data collected from neurologist and brain tumor patients. Neural network is trained using generated dataset to identification epilepsy seizure is due to brain tumor or other neural disorders. Fig.6. shows flow of brain tumor epilepsy ANN classifier network formation. Fig.7. shows the testing flow of brain tumor epilepsy ANN classifier.

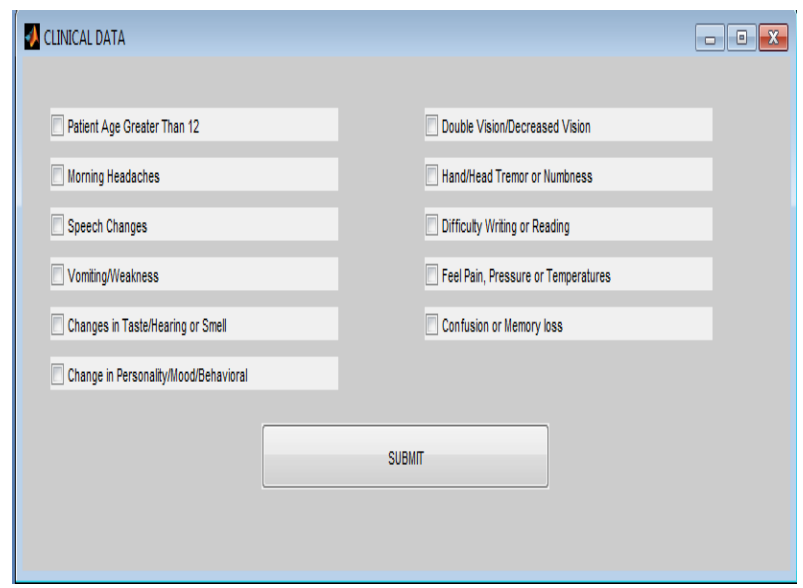

Figure 5: Clinical data questions 


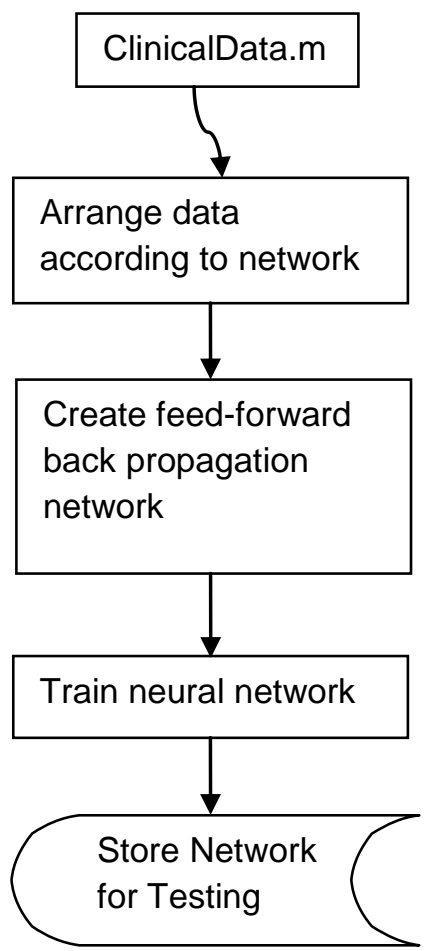

Figure 6: Flow of Brain tumor epilepsy ANN classifier network formation

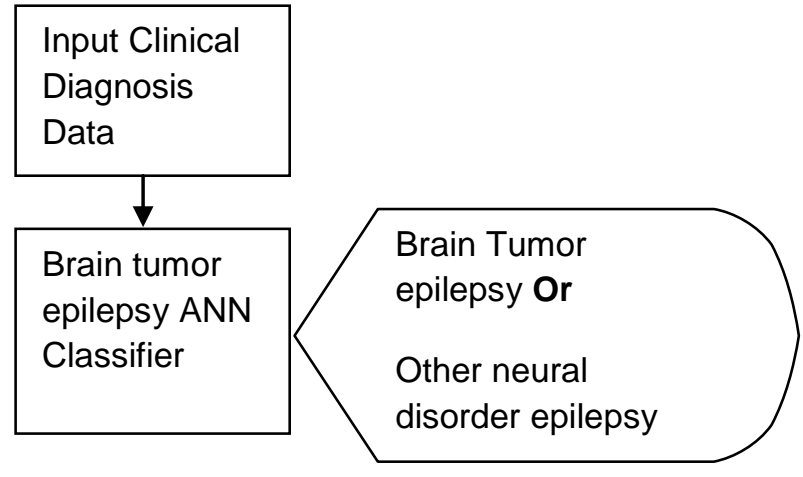

Figure 7: Testing flow of Brain tumor epilepsy ANN classifier

\section{RESULT AND DISCUSSIONS}

The proposed technique for brain tumor epilepsy seizure detection is implemented using MATLAB 7.11 on windows 7 PC with Intel 17 processor. Here, the wavelet level was chosen as 5 for extracting the feature of the signal and for ApE calculation, window length $\mathrm{m}=4$ and tolerance $\mathrm{rr}=5$ is considered. The hidden layer neuron was set as 20 .
Performances of the implemented method is measured based on performance indices such as sensitivity, specificity, precision and accuracy parameters. The true positive, true negative, false positive and false negative values are calculated from the results obtained. The above four values are used to calculate performance indices as specified in the equations given below. Table 1 gives the performance evaluation for brain tumor epilepsy seizure. Fig.5 shows the GUI of the proposed system.

Specificity: Number of correctly detected negative patterns/total number of actual negative patterns. A negative pattern indicates a detected normal/non-seizure.

Specificity $=\frac{\mathrm{TN}}{(\mathrm{FP}+\mathrm{TN})}$

Sensitivity: Number of correctly detected positive patterns/total number of actual positive patterns. A positive pattern indicates a detected seizure.

Sensitiviy $=\frac{T P}{(T P+F N)}$

Accuracy: Number of correctly classified patterns/total number of patterns..

$$
\begin{aligned}
& \text { Accuracy }=\frac{\mathrm{TP}+\mathrm{TN}}{\mathrm{TP}+\mathrm{FP}+\mathrm{TN}+\mathrm{FN}} \\
& \text { Precision }=\frac{\mathrm{TP}}{(\mathrm{TP}+\mathrm{FP})}
\end{aligned}
$$

Table 1. Performance Evaluation Table for Brain Tumor Epilepsy identification

\begin{tabular}{|l|l|}
\hline \multicolumn{1}{|c|}{ Parameter } & $\begin{array}{c}\text { Proposed } \\
\text { Method }\end{array}$ \\
\hline Sensitivity & 88 \\
\hline Specificity & 89 \\
\hline Accuracy & 93 \\
\hline Precision & 90 \\
\hline
\end{tabular}




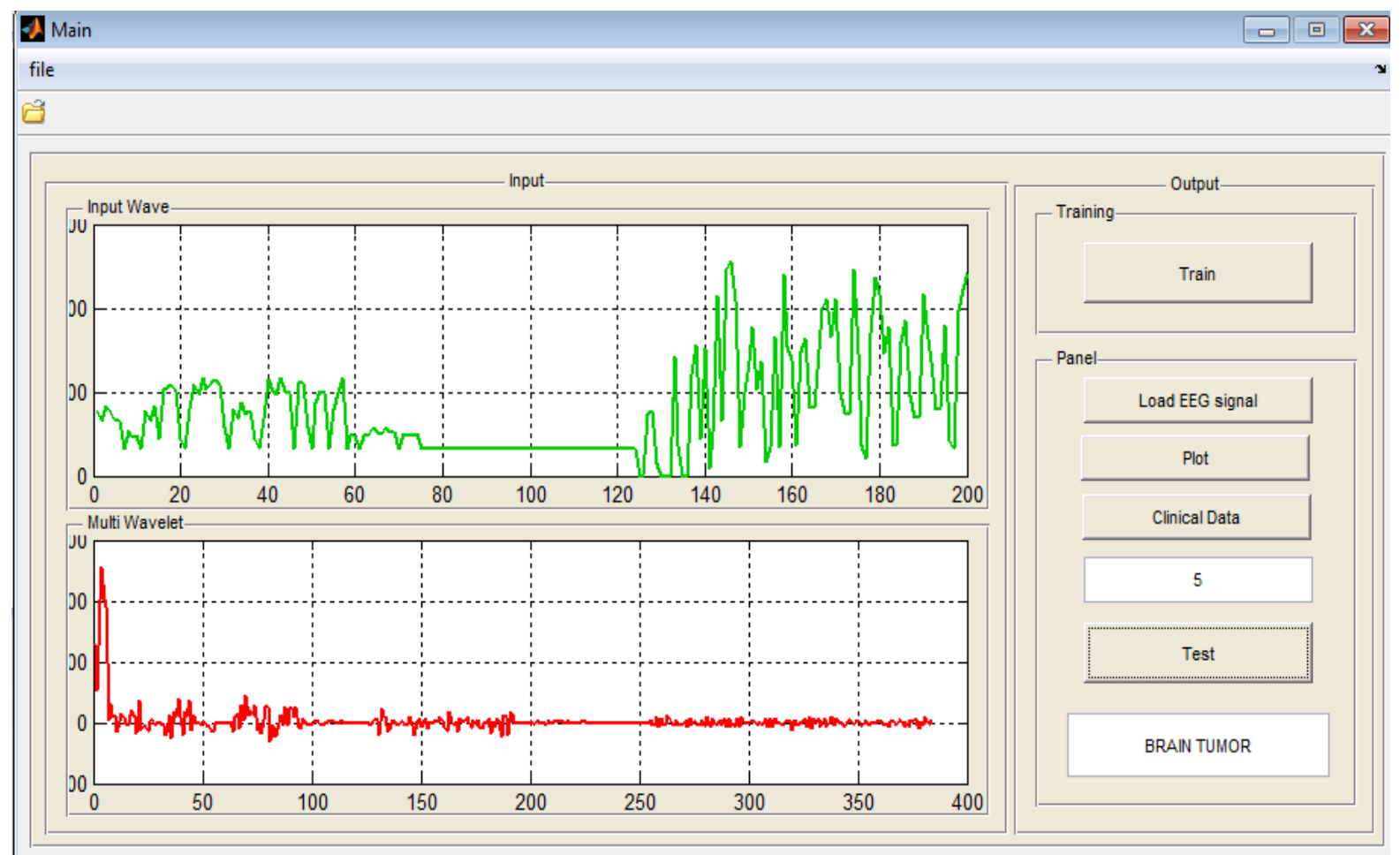

Figure 5: GUI of proposed method system

\section{CONCLUSION}

This paper implemented a hybrid system that combines biomedical engineering techniques and clinical diagnosis data in a multistage scheme for detection of brain tumor epilepsy seizure. In a two level identification technique, first level classify the given EEG signal in to normal and epilepsy seizure, based on the first level input second level identifies the epilepsy seizure is from brain tumor or other neural disorders using clinical diagnosis data. The algorithm used (MWT, FFNN and ApN) by the module are well proven in the area of biomedical signal processing. The system consists of an attractive and informative GUI. The technique is implemented and tested on data of 150 EEG signals. Results are promising with classification accuracy of $93 \%$. Work is in progress to make system more robust by testing with more brain tumor epilepsy seizure EEG signals data.

\section{REFERENCES}

[1] J.D. Bronzino, "Biomedical Engineering Handbook", New York: CRC Press LLC, Vol. I, 2nd edition, 2000.

[2] American Brain Tumor Association "Brain Tumor Primer - A Comprehensive Introduction to Brain Tumor", 9th edition, Available online. Last accessed September 24, 2012.

[3] Atlanta, Ga: "American Cancer Society: Cancer Facts and Figures 2012", American Cancer Society 2012. Available online. Last accessed September 24, 2012.

[4] Edward B Bromfield and Selim R Benbadis "EEG in Brain Tumors", Medscape Reference. Available online. Last accessed September 25, 2012.

[5] Selim R Benbadis "Encephalopathic EEG Patterns", Medscape Reference. Available online. Last accessed September 26, 2012.
[6] http://www.fil.ion.ucl.ac.uk/EEGvolunteerguide.pdf

[7] http://www.brain-surgery.com/primer.html

[8] Sharanreddy and Dr.P.K.Kulkarni "Review of Significant Research on EEG based Automated Detection of Epilepsy Seizures \& Brain Tumor", International Journal of Scientific \& Engineering Research, Volume 2, Issue 8, Aug-2011, ISSN 22295518.

[9] Sivasankari N and K. Thanushkodi, "Automated Epileptic Seizure Detection in EEG Signals Using Fast ICA and Neural Network", Int. J. Advance. Soft Comput. Appl., Vol.1, No.2, pp.91-104, November 2009.

[10] Ling Guo, Daniel Rivero and Alejandro Pazos, "Epileptic seizure detection using multiwavelet transform based approximate entropy and artificial neural networks", Journal of Neuroscience Methods, Vol.193, pp.156-163, 2010.

[11] Kifah Tout, Nisrine Sinno and Mohamad Mikati, "Prediction of the Epileptic Events 'Epileptic Seizures' by Neural Networks and Expert Systems", International Journal of Biological and Medical Sciences, Vol.5, No.1, pp.38-45, 2010

[12] Laxman Tawade and Hemant Warpe, "Detection of Epilepsy Disorder Using Discrete Wavelet Transforms Using MATLABs", International Journal of Advanced Science and Technology, Vol.28, pp.17-24, March 2011.

[13] The testing dataset is referred from the link: http://physionet.fri.uni-lj.si/pn6/chbmit/.

[14] Ali Shoeb and john Guttag "Application of Machine Learning To Epileptic Seizure Detection" Proceedings of the 27th International Conference on Machine Learning 
(ICML-10), June 21-24, 2010, Haifa, Israel. Omnipress 2010, ISBN 978-1-60558-907-7.

[15] M. Murugesan and Dr. (Mrs.).R. Sukanesh "Towards Detection of Brain Tumor in Electroencephalogram Signals Using Support Vector Machines" International Journal of Computer Theory and Engineering, Vol. 1, No. 5, December, 2009.

[16] Sharanreddy and Dr.P.K.Kulkarni "Literature Survey on EEG based Automatic Diagnosis of Epilepsy seizures \& Brain Tumor using WT and ANN" International Conference on Biomedical Engineering (ICBME 2011), Dec 10-12, 2011, Manipal, India.

[17] Sharanreddy and Dr.P.K.Kulkarni "Necessity for Automated Analysis of EEG Signal for Detection of Multiple Neurological Disorders" International Conference on Evolutionary Trends in Information Technology (ICETIT 2012), Sep 15-17, 2012, VTU Belgaum, India.

[18] Sharanreddy and Dr.P.K.Kulkarni "An Improved Approximate Entropy Based Epilepsy Seizure Detection Using Multi-Wavelet and Artificial Neural Networks" International Journal of Biomedical Engineering and Technology, Accepted - 22 Jan 2013, InderScience Publishers, UK, ISSN online: 1752-6426, ISSN print: 1752-6418.

[19] Sharanreddy and Dr.P.K.Kulkarni "Multi-Wavelet Transform Based Epilepsy Seizure Detection" Proceedings - 2012 IEEE EMBS Conference on Bio Engineering \& Sciences (IECBES 2012) Dec.17-19, 2012, Langkawi, Malaysia. ISBN - 978-1-4673-1666-8.

[20] Sharanreddy and Dr.P.K.Kulkarni "Detection of primary brain tumor present in eeg signal using wavelet transform and neural network" International Journal of Biological and Medical Research, 2013; 4(1): ISSN 2855-2859.

[21] Seenwasen Chetty, Ganesh K. Venayagamoorthy "An investigation into the Detection of brain tumours using electroencephalography (EEG) signals with Artificial neural networks" Computational Intelligence Group, Department of Electronic Engineering M L Sultan Technikon.

[22] Habl, M. and Bauer, Ch. and Ziegaus, Ch., Lang, Elmar and Schulmeyer, F. "Can ICA help identify brain tumor related EEG signals?” Proceedings / ICA 2000, Second
International Workshop on Independent Component Analysis and Blind Signal Separation: Helsinki, Finland, June 19 - 22, 2000. Unspecified, pp. 609-614. ISBN 95122-5017-9.

[23] Small, Joyce Graham Bagchi, Basu K.Kooi, Kenneth A. "Electro-clinical profile of 117 deep cerebral tumors" Elsevier Inc Electroencephalography and Clinical Neurophysiology, Vol 13, Issue 2 , Pages 193-207, April 1961

[24] Forrest Sheng Bao, Donald Yu-Chun Lie, and Yuanlin Zhang, "A New Approach to Automated Epileptic Diagnosis Using EEG and Probabilistic Neural Network", in Proceedings of the 2008 20th IEEE International Conference on Tools with Artificial Intelligence, Vol. 02, pp. 482-486, 2008.

[25] Steven Walczak and William J. Nowack, "An Artificial Neural Network Approach to Diagnosing Epilepsy Using Lateralized Bursts of Theta EEGs", Journal of Medical Systems, Vol. 25, No. 1, pp. 9-20, February 2001

[26] Maan M. Shaker, "EEG Waves Classifier using Wavelet Transform and Fourier Transform", International Journal of Biomedical Sciences, Vol 1, No 2, 2006, ISSN 13061216.

[27] Nick Yeung, Rafal Bogacz, Clay B. Holroyd and Jonathan D. Cohen "Detection of synchronized oscillations in the electroencephalogram: An evaluation of methods" Psychophysiology, 41 (2004), 822-832. Blackwell Publishing Inc. Printed in the USA.

[28] Samhita P, Venkataraman V, Radhakrishnan, Kurupath Radhakrishnan, Ravi M, and Sankara P. "Electro-clinical characteristics and postoperative outcome of medically refractory tumoral temporal lobe epilepsy" Vol 53, Issue 1, Neurology of India, March 2005.

[29] Young Zoon Kim, Eun Hee Lee and Kyoung Soo Lee "Clinical Analysis for Brain Tumor-Related Epilepsy during Chemotherapy for Systemic Cancer with Single Brain Metastasis" Cancer Res Treat. 2011; 43(3):160 169.

[30] M. Gelabert-González*, J.M. Santín Amo, A. Arcos Algaba, R. Serramito García "Intracranial gangliogliomas. A review of a series of 20 patients", Sociedad Espa nola de Neurología. Published by Elsevier España, 2010. 